\title{
Numerical Modelling and Materials Characterisation for Integrated Micro Electro Mechanical Systems
}

\author{
Henry Baltes, Jan G. Korvink and Oliver Paul \\ Physical Electronics Laboratory, \\ ETH Zurich, CH-8093 Zurich, Switzerland \\ Email: baltes@iqe.phys.ethz.ch
}

\begin{abstract}
Integrated micro electro mechanjcal systems (iMEMS) include sensors, actuators and circuits made by silicon IC technology combined with micromachining, deposition or electroplating. We present two essential iMEMS development tools:

(i) the data base ICMAT of material parameters obtained from measuring process-dependent IC thin film electrical, magnetic, thermal and mechanical properties by using dedicated materials characterisation microstructures and (ii) the toolbox SOLIDIS, providing coupled numerical modelling of the electrical, magnetic, thermal and mechanical phenomena and their boundary and interface conditions occurring in $i M E M S$ devices in a uniform and consistent environment.
\end{abstract}

\section{Introduction}

Micro Electro Mechanical Systems (MEMS) include micromachined structures with mechanical, thermal, magnetic and fluid effects for sensors and actuators. Integrated Micro Electro Mechanical Systems (iMEMS) combine MEMS with integrated circuits. Our approach to $i M E M S$ is to combine industrial CMOS or bipolar IC technology with bulk or surface micromachining, thin film deposition or electroplating [1]. Integrated thermoelectric, magnetic, mechanical and chemical microtransducers have been demonstrated in this way [2]-[7].

Design and simulation of $i M E M S$ devices requires precise knowledge of process-dependent material parameters, such as resistivity and its temperature coefficient, thermal conductivity, heat capacity, Seebeck coefficient, stress, Young's modulus, and Poisson's ratio of all IC layers (which include the metallisation and polysilicon layers, the field, contact and via oxides, the standard passivation) and any additional layers such as silicon nitride passivation for sensors. In Section 2, we report how these material properties are measured for a variety of industrial CMOS IC processes by designing material characterisation microstructures fabricated by the corresponding IC process.

In efficient $i M E M S$ devices, magnetic, thermal or mechanical vector fields couple strongly (and often nonlinearly) to "ordinary" IC electrical properties. Thus iMEMS 
simulation goes beyond semiconductor device modelling based on some approximation of Boltzmann's equation [8]. In Section 3 we report the $i M E M S$ simulation toolbox SOLIDIS, which includes equations describing the various reversible and irreversible transducer effects and their coupling [9]. Specific simulation results are reported for a thermoelectric characterisation device, a CMOS micromirror and a micromagnetic flux concentrator. There is a "bootstrap" connecting Section 2 and 3: Modelling allows the validation of the materials characterisation microstructures. In turn, the simulation can be validated by comparison with the measured material properties. The latter serve as input parameters for realistic iMEMS device simulation and optimisation.

\section{IC Material Properties}

Thermal properties of IC thin films depend on their fabrication process. The layers being characterised must be produced with the same process as the corresponding microdevice layer. We therefore use characterisation microstructures fabricated with the IC process used for the fabrication of microtransducers [10]. Such IC compatible microstructures are smaller than $500 \mu \mathrm{m}$ and have been successfully used for the determination of thermal conductivities [11, 12] and the heat capacities [13] of CMOS layers, and of transport coefficients of CMOS polysilicon [14], such as its Seebeck coefficient.

IC compatible characterisation structures exploit ideas implemented in microsensors, such as micromachined dielectric beams and integrated CMOS polysilicon resistors and thermistors. In contrast to sensors optimised to transduce an external signal into the electrical domain, the structures have to sense a property of one of their constitutive materials. Cross-sensitivities to other properties are suppressed by suitable design optimisation. Validation and optimisation of a thermal structure with numerical simulation is described in [15]. A further case is detailed in Section 3.2.

\subsection{Thermal and Electronic Properties of CMOS Layers}

As an example we describe the measurement of the Seebeck coefficient of CMOS polysilicon, a parameter of thermoelectric CMOS sensors [1]. The test structure is shown schematically in Figure 1. It consists of a micromachined cantilever and lateral arms composed of the sandwich of dielectric CMOS layers. Micromachining is made possible by suitable layout design of the field, contact, via, and pad masks. Cuts through the corresponding layers allow the silicon to be anisotropically etched away and the structure to be undercut. A micrograph is shown in Figure 2.

At its tip the cantilever contains two CMOS polysilicon resistors. The one closer to the end serves as a heater. The other resistor is used as a thermistor to determine the temperature at the free end. A rectangular polysilicon film to be characterised is integrated into the dielectric sandwich between the hot cantilever tip and the bulk silicon die. The polysilicon is contacted at its hot and cold ends with the lower CMOS metal. When electrical power is dissipated in the heater, a thermoelectric voltage $U_{t h}=\alpha \Delta T$ is measured between these two contacts, where $\alpha$ is the Seebeck coefficient sought and $\Delta T$ is the temperature difference between the hot and the cold contacts of the polysilicon. The electrical measurement of $U_{t h}$ is straightforward. For the accurate determination of $\Delta T$ we improved the geometry of earlier versions of the test structure [14]. One improvement is the integration of a temperature smoothing CMOS metal layer, that extends the hot polysilicon contact beyond the temperature monitoring resistor. With this test structure we have shown that the 
Seebeck coefficient of the standard gate poly of three commercial CMOS processes is in the range between 108 and $120 \mu \mathrm{V} / \mathrm{K}[11 \mathrm{i}$

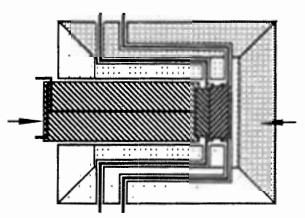

E.2. Etched cavity

$\square$ Dielectrics

Poly heaters and temperature sensors

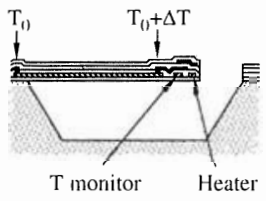

Silicon substrate

Poly layer

Metal layers

ㄹ Metal lead

Figure 1. Schematic top view (left) and cross-section (right) of CMOS characterisation structure to determine the Seebeck coefficient of the gate or capacitor polysilicon for CMOS mucrosensors. The Seebeck coefficient is measured between $T_{o}$ and $T_{o}+\Delta T$.

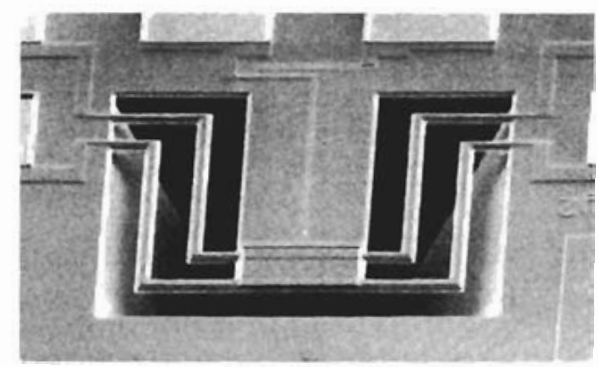

Figure 2. SEM micrograph of the micromachined Seebeck test structure. Heater and temperature monitoring resistors are integrated between the lateral connection arms.

\begin{tabular}{|l|l|l|l|l|}
\hline \hline $\begin{array}{l}\text { Property } \\
\text { Layer }\end{array}$ & $\begin{array}{l}\rho_{s q} \\
\left(10^{-3} \Omega \mathrm{cm}\right)\end{array}$ & $\begin{array}{l}\text { TC of } \rho \\
\left(p p m K^{-1}\right)\end{array}$ & $\begin{array}{l}\alpha-\alpha_{M e t 1} \\
(\mu V / K)\end{array}$ & $\begin{array}{l}\kappa_{t h} \\
(W / m K)\end{array}$ \\
\hline$n^{+}$-Poly & $25 \pm 1$ & $860 \pm 30$ & $-120 \pm 5$ & $24 \pm 1.5$ \\
\hline$p^{+}$-Poly & $215 \pm 5$ & $-140 \pm 5$ & $190 \pm 10$ & $17 \pm 1.5$ \\
\hline Metal 1 & $65 \pm 0.7$ & $2960 \pm 100$ & 0 & $181 \pm 8$ \\
\hline Metal 2 & $36 \pm 0.4$ & $3010 \pm 100$ & & $166 \pm 8$ \\
\hline
\end{tabular}

Table I. Sheet resistance $\rho$, temperature coefficient of $\rho$, Seebeck coefficient $\alpha$ and thermal conductivity $\kappa_{t h}$ of conducting layers of the $1.2 \mu \mathrm{m}$ CMOS process of Austria Mikrosysteme (AMS) at $300 \mathrm{~K}$.

\begin{tabular}{|l|l|l|}
\hline \hline $\begin{array}{l}\text { Property } \\
\text { Layer }\end{array}$ & $\begin{array}{l}\kappa_{\text {th }} \\
(W / m K)\end{array}$ & $\begin{array}{l}c \\
\left(\mathrm{Jcm}^{-3} K^{-1}\right.\end{array}$ \\
\hline Field oxide & $1.28 \pm 0.18$ & \multirow{2}{*}{$1.05 \pm 0.1$} \\
\cline { 1 - 2 } Contact ox. & $1.32 \pm 0.18$ & \multirow{2}{*}{$0.16 \pm 0.24$} \\
\cline { 1 - 2 } Via oxide & $1.16 \pm 0.7 \pm 0.25$ \\
\hline Passivation & $1.5 \pm 0.25$ & 2.7 \\
\hline
\end{tabular}

Table II. Thermal conductivity $\kappa_{t h}$ and heat capacity $c$ of dielectric thrn films of the AMS $1.2 \mu \mathrm{m}$ CMOS process at $300 \mathrm{~K}$.

Similar thermal test structures provide the thermal conductivities and heat capacities of CMOS layers. Sheet, resistances and temperature coefficients (TC) of resistance are measured with van-der-Pauw structures. In our laboratory, measurements of material properties are systematically performed between 100 and $420 \mathrm{~K}$ to obtain temperature coefficients and to allow better understanding of the process dependence of the properties. Experimental thermal and electronic properties of films of the CMOS process of EM Microelectronic Marin, Austria Mikrosysteme (AMS) and European Silicon Structures (ES2) were measured. As an example, results of AMS $1.2 \mu \mathrm{m}$ CMOS 
process are shown in Tables I and II. The thermal conductivities of polysilicon and metal layers deviate strongly from bulk values. Such deviations show the importance of characterising thermal material properties relevant for $i M E M S$.

\begin{tabular}{|l|l|l|l|}
\hline \hline Thin film & $\sigma(\mathrm{MPa})$ & $E(\mathrm{GPa})$ & $\nu$ \\
\hline LPCVD silicon nitride & $1040 \pm 30$ & $195 \pm 6$ & $0.13 \pm 0.02$ \\
\hline Contact oxide & $-40 \pm 10$ & $20 \pm 10$ & $0.5_{-0.1}^{+0.0}$ \\
\hline $\begin{array}{l}\text { Intermetal dielectric standard } \\
\text { passivation }\end{array}$ & $-37 \pm 6$ & $65 \pm 5$ & $0.2 \pm 0.2$ \\
\hline $\begin{array}{l}\text { PECVD silicon nitride passiva- } \\
\text { tion for CMOS sensors }\end{array}$ & $82 \pm 5$ & $97 \pm 6$ & $0.13 \pm 0.07$ \\
\hline \hline
\end{tabular}

Table III. Stress $\sigma$, Young's modulus $E$ and Poisson's ration $\nu$ of the dielectric layers of the $2 \mu \mathrm{m}$ CMOS process of EM Microelectronic Marin, used for the fabrication of microsensors and actuators.

\subsection{Mechanical Properties of CMOS Layers}

Mechanical thin film properties of the $2 \mu \mathrm{m}$ CMOS process of EM Microelectronic Marin (EM), Switzerland, were determined using the bulge test. This method has previously been applied to measure the residual stress $\sigma$ and the elastic modulus $E$ of polymer films $[16,17]$, and of CVD silicon nitride and polysilicon [18]. It exploits the load-deflection behavior of thin film membranes, as shown in Figure 3. The mid-point deflection $W$ of thin membranes under unilateral pressure $P$ is described by

$$
P=C_{1} \sum_{i} \frac{h_{i}}{a^{2}} \sigma_{i} W+C_{2} \sum_{i} f\left(\nu_{i}\right) \frac{h_{i}}{a^{4}} \frac{E_{i}}{1-\nu_{i}} W^{3},
$$

where $h_{i}, \sigma_{i}, \nu_{i}$, and $E_{i}$ denote the thicknesses, the stresses, the Poisson's ratios and the elastic moduli, respectively, of the membrane component layers, and $a$ denotes a linear dimension of the membrane. The constants $C_{1}$ and $C_{2}$ and the function $f(\nu)$ for square, rectangular, and circular membranes have been calculated with finite element and analytical methods [19]. Implicit in the equation is the assumption that the contribution of membrane bending to the total energy is negligible. This condition is fulfilled by sufficiently thin and tensile membranes.

We produced membranes containing the layers to be characterised using the standard CVD deposition conditions of the CMOS process. The membranes are composed of a $1680 \AA$ thick, tensile base layer of LPCVD silicon nitride, on top of which standard thickness laysers of either the contact oxide, the intermetal oxide, or a $2.08 \mu \mathrm{m}$ thick nitride passivation layer optimised for CMOS microsensors were deposited [2]. After the CVD steps, rectangular membranes with sizes up to $2 \mathrm{~mm}$ and side ratios of $1: 1,1: 2$, and 1:4 were produced by silicon micromachining in $\mathrm{KOH}$. During the etch the wafer-fronts were mechanically protected. Pressure-dependent deflections of the membranes were measured with a contactless surface profiler.

Figure 4 shows the mid-point responses of various LPCVD nitride membranes with a side ratio of 1:4. First the silicon nitride properties were obtained with single layer membranes. Based on these values, the mechanical properties of the second thin film in the bilayer membranes were then determined. Results are listed in Table III. The contact and intermetal dielectrics are under compressive stress, whereas the nitride layers are under tension. Poisson's ratios of both nitrides and the contact oxide show 
a significant deviation from the values of 0.25 to 0.3 usually assumed in the literature. Clearly, the determination of Poisson's ratio of thin films deserves more attention than it has hitherto received.
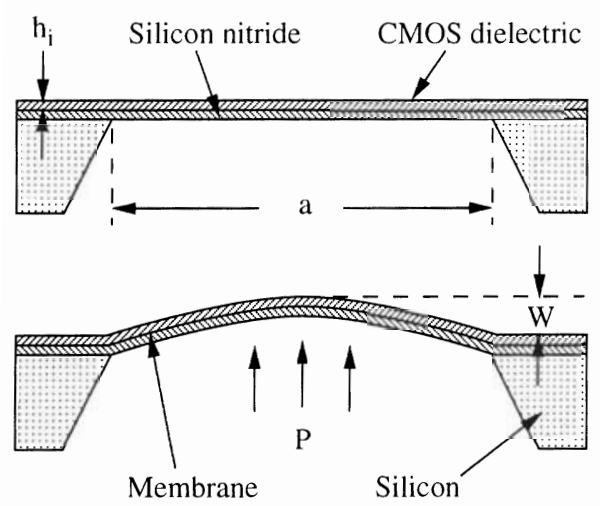

Figure 3. Micromechanical membrane to determine mechanical thin film parameters (a) After fabrication and micromachining, (b) Deflection under pressure load.

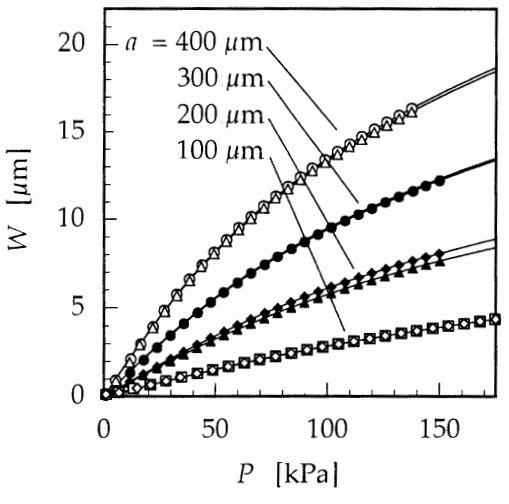

Figure 4. Deflection of rectangular membranes with side length ratio 1:4 as a function of applied pressure. The membranes consist of $1680 \AA$ silicon nitride.

\section{3. iMEMS Modelling and Simulation}

Sensor and actuator development using industrial IC processes places tight limits on device performance. Modelling and simulation is important for the purposes of device optimisation, and understanding the detailed device operation principles. Simulations must be representative of the physical processes and provide sufficiently accurate predictions of sensitivity. High performance simulation requires three key elements: (i) precise knowledge of material properties and device geometries, (ii) accurate (selfcorrecting) numerical solution methods and (iii) correct model systems (physical model equations, geometry, boundary and interface conditions). The operation of $i M E M S$ is governed by one or more of a large variety of coupled physical effects. A functional tool establishes consistent solutions, and supports extensions when required [9]. With these prerequisites, simulation significantly reduces the number of prototypes required for the development process.

\subsection{The $i M E M S$ Simulation Toolbox SOLIDIS}

SOLIDIS addresses the simulation of coupled effects that arise in sensor chips and in packaged solid-state sensors and actuators. It comprises a set of computational kernels and interactive visualisation front ends. Each kernel implements a hybrid finite element discretisation [20] of a tailored set of partial differential equations. A scalar field $\psi(\vec{x})$ and its associated vector field $\vec{\Phi}(\vec{x})$ are separately approximated over the domain covered by a mesh of finite elements and nodes:

$$
\psi(\vec{x})=\sum_{i=1}^{n} p_{i} S_{i}(\vec{x}) ; \quad \vec{\Phi}(\vec{x})=\sum_{j=1}^{m} f_{j} \vec{V}_{j}(\vec{x}) .
$$


The subscripts are associated with mesh nodes. The $S_{i}(\vec{x})$ are the scalar basis functions and $\vec{V}_{j}(\vec{x})$ are vector basis functions. The $p_{i}$ and $f_{i}$ are scalar weights. For an equation of the form $\vec{\nabla} \cdot \vec{\Phi}=\rho(\vec{x})$, with $\vec{\Phi}=\beta(\vec{x}) \vec{\nabla} \psi$, where $\rho(\vec{x})$ and $\beta(\vec{x})$ are parameters ${ }^{1}$, we write two weighted residual equations, each integrated over the simulation domain $\Omega$,

$$
\int_{\Omega} \vec{V}_{i} \cdot[\vec{\Phi}-\beta \vec{\nabla} \psi] d \Omega=0 ; \quad \int_{\Omega} S_{\imath}[\vec{\nabla} \cdot \vec{\Phi}-\rho] d \Omega=0 .
$$

After substitution of the expansions for $\psi$ and $\vec{\Phi}$ and some standard manipulations we obtain two discrete matrix residual equations $A f+B p=0$ and $B^{T} f-r=0$. After eliminating $f$ we obtain $B^{T} A^{-1} B p+r=0$. This is the equation to be assembled and solved for the nodal degrees of freedom $p$, from which $f$ is computed. This hybrid method ensures better compatibility between $\psi$ and $\vec{\Phi}$ than with a conventional finite element method, and bears fruit when used in conjunction with mesh adaptivity converging to spatially accurate solutions, and when discretising coupled effects that require accurate distributions of $\vec{\Phi}$.

\subsection{Characterisation Device Modelling}

We next consider a device for measuring the Seebeck coefficient of a BiCMOS polysilicon layer, as described in Section 2.1 and Figure 2. One modelling goal is to minimise external influences and stray effects. Accurately resolved field distributions and fluxes in $3 \mathrm{D}$ are therefore essential.

For the simulation we model half of the geometry of the beam structure, taking advantage of the beam's symmetrical layout (Figure 5). The device is operated in vacuum. Its small size ensures negligible heat loss through radiation. The thermal conductivities are taken from Tables I and II. The power dissipated is accounted for in all conductors with resistivities taken from Table I.

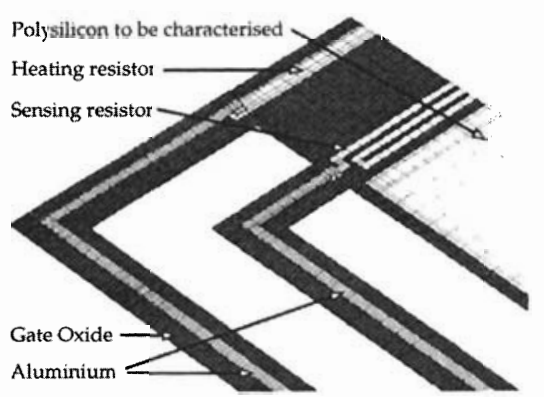

Figure 5. Part of the 3D mesh used for the modelling. The passivation, intermetal oxide, contact oxide, and the aluminium covering the heater and temperature sensor, is removed for clarity. The mesh has 15,869 finite elements and 20,112 nodes.

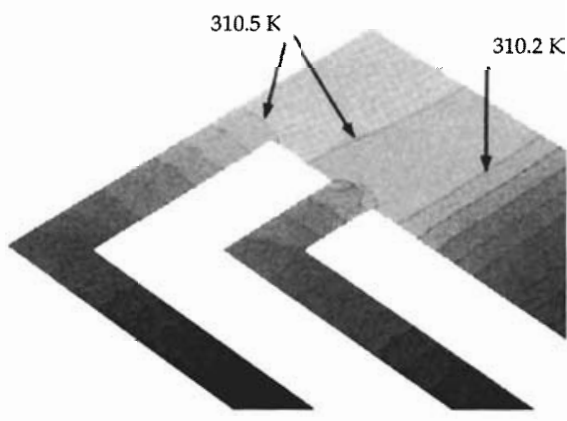

Figure 6. The temperature contours on the upper surface of the mesh depicted in Figures 5. $\delta T$ between contours is $0.3 \mathrm{~K}$. On the polysilicon $T_{h}=310.2 \mathrm{~K}$ and $T_{o}=300 K$. Note the uniform temperature distribution over the temperature sensor.

\footnotetext{
${ }^{1}$ Note that we are not restricted to this particular partial differential equation.
} 
Modelling was used to verify the device function, and quantifyd stray effects arising from the current-carrying arms connected to the free end of the beam. Figure 6 indicates the effectiveness of the aluminium sheets in providing a low-resistance path for the generated heat into the polysilicon beam, and for averaging the temperature profile over the meandering thermometer. The computed temperature drop over the beam is $10.2 \mathrm{~K}$. The resistor is only $0.12 \mathrm{~K}$ hotter than the hot end of the polysilicon, and uniform to within $0.05 \mathrm{~K}$. Thus all assumptions regarding the temperature measurement (see Section 2.1) hold.

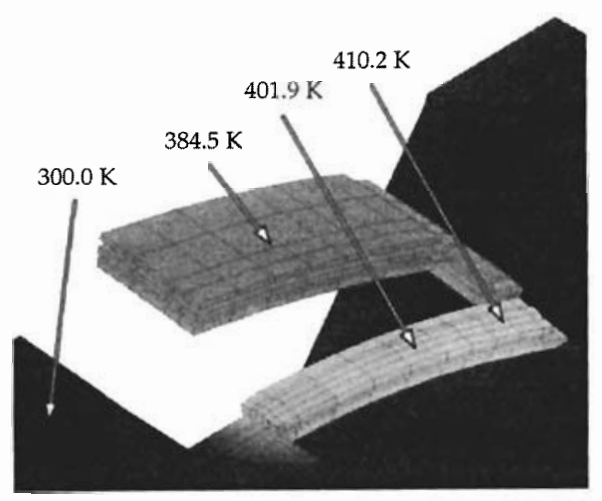

Figure 7. The mesh of the integrated micromirror, with the temperature distribution over the surface (most oxides and nitrides are removed) and in the mechanically deformed $(\times 30)$ state.

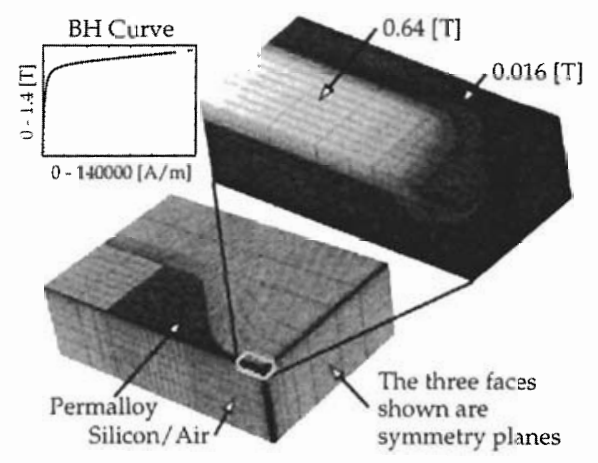

Figure 8. The mesh of $1 / 8$ of the integrated microconcentrator (dark shading). Above right is the magnetic induction flux distribution over the tip of the magnetoconcentrator. Above left is the measured BH curve of the permalloy.

\subsection{Sensor and Actuator Modelling}

In the thermomechanical micromirror actuator [6], Joule heat is dissipated in a polysilicon resistor within the mirror's suspension beams to generate a local temperature rise. This results in thermal expansion of the beam's materials. The difference in thermal expansion coefficients causes bending of the beam (also called the multimorph effect) and hence deflection of the mirror. A principal target is to obtain maximum mirror deflection for given input power. It is also essential that the mirror does not warp during actuation (see Figure 7). Finally, effects due to mask misalignment - causing asymmetry in the deflection - must be minimised. The simulations rely heavily on knowing the thermoelectric and mechanical properties listed in Tables I, II and III accurately. A full study is presented in [21].

For the integrated magnetic flux concentrator [3] in Figure 8, a thin layer $(5 \mu \mathrm{m})$ of permalloy is electroplated onto the surface of a chip and serves to focus the magnetic flux onto a magnetotransistor. The saturative nature of the permalloy is a source of nonlinearity. Saturation is a local effect - the concentrator causes large variations in magnetic field intensity. A coarse mesh of 20,000 finite elements underestimates the concentrated flux density and smoothens the saturation curve of the device. Numerically, large finite elements are driven uniformly into saturation, whereas this should only happen for a small sub-region. Adaptive mesh refinement leads to meshes in the region of 80,000 finite elements. A full numerical study is presented in [22]. 


\section{Conclusions}

In this paper we demonstrated a variety of characterisation microstructures for measuring process-dependent electrical, thermal and mechanical properties of IC layers used in $i M E M S$ devices. The resulting data base ICMAT in combination with the simulation toolbox SOLIDIS, also presented here, allows precise and efficient numerical modelling of a wide variety of $i M E M S$ devices and thus provides a crucial tool for resource-efficient prototyping.

Future measurements will aim at additional coupling parameters, e. g. thermomechanical and piezoresitive coefficients. The extension to further CMOS processes involved in $i M E M S$ manufacturing is another obvious task. This will lead to a set of standard characterisation microstructures to be added to the conventional IC process test structures. Likewise, modelling will be extended to further coupling effects such as convection, the piezo-electric coupling and the Peltier effect. It is imperative that the mutual validation of characterisation structures and modelling tools is maintained for any reliable extension of ICMAT and SOLIDIS.

\section{Acknowledgements}

It is our pleasure to thank Dr. Arokia Nathan, visiting Professor at ETH Zurich, on leave from the University of Waterloo, Ontario, Canada, for enlightening discussions.

\section{References}

[1] H. Baltes, O. Brand, J. G. Korvink, R. Lenggenhager, O. Paul, "IMEMS - integrated micro electro mechanical systems by VLSI and micromachining," ESSDERC'94 Proc. 24th European Solid State Device Research Conference, (Editions Frontires, Gif-sur-Yvette, France), pp. 273-280 (1994).

[2] R. Lenggenhager, D. Jaeggi, P. Malcovati, H. Duran, H. Baltes, E. Doering, "CMOS membrane infrared sensor and improved TMAHW etchant," IEDM Technical Digest, (IEEE), pp. 531-534 (1994).

[3] M. Schneider, R. Castagnetti, M. G. Allen, H. Baltes, "Integrated flux concentrator improves CMOS magnetotransistor," Proc. IEEE MEMS, pp. 151-156 (1995).

[4] M. Hornung, R. Frey, O. Brand, H. Baltes, C. Hafner, "Ultrasound barrier based on packaged micromachined membrane resonators," Proc. IEEE MEMS, pp. 151$156(1995)$.

[5] O. Paul, A. Häberli, P. Malcovati, H. Baltes, "Novel integrated thermal pressure gauge and read-out circuit by CMOS IC design," IEDM Technical Digest, (IEEE), pp. 131-134 (1994).

[6] J. Bühler, H. Baltes, "Thermally actuated CMOS micro mirrors," Sensors and Actuators 47, pp. 525-575 (1995).

[7] F.-P. Steiner, A. Hierlemann, C. Cornila, G. Noetzel, M. Bächtold, J. G. Korvink, W. Göpel, H. Baltes, "Polymer coated capacitive microintegrated gas sensor," Transducers'95 Digest of Technical Papers, in press (1995).

[8] S. Selberherr, "Analysis and Simulation of Semiconductor Devices," (SpringerVerlag, Wien) (1984).

[9] J. G. Korvink, J. Funk, H. Baltes, "IMEMS modelling," Sensors and Materials 6, pp. 235-243 (1994). 
[10] O. Paul, M. von Arx and H. Baltes, "Process-dependent thermophysical properties of CMOS IC thin films," Transducers'95 Digest of Technical Papers, in press $(1995)$.

[11] F. Völklein and H. Baltes, "A microstructure for measurement of thermal conductivity of polysilicon thin films," J. of Microelectromech. Systems, 1, pp. 193-196 (1993).

[12] O. Paul, M. von Arx, and H. Baltes, "CMOS IC layers: complete set of thermal conductivities," Proc. Intl. Workshop on Semiconductor Characterization, (NIST, Gaithersburg), in press (1995).

[13] M. von Arx, O. Paul, H. Baltes, "Determination of the heat capacity of CMOS layers for optimal CMOS sensor design," Sensors and Actuators A, 47, pp. 428-431 (1995).

[14] O. Paul and H. Baltes, "Measuring thermogalvanomagnetic properties of polysilicon for the optimization of CMOS sensors," Transducers'93 Digest of Technical Papers (IEE, Japan, Tokyo), pp. 606-609 (1993).

[15] O. Paul, J. Korvink and H. Baltes, "Determination of the thermal conductivity of CMOS IC polysilicon," Sensors and Actuators A, 41-42, pp. 161-164 (1994).

[16] P. Lin, "The in-situ measurement of biaxial modulus and residual stress of multilayer polymeric thin films," Mat. Res. Soc. Symp. Proc., 188, pp. 41-46 (1990).

[17] M. G. Allen, M. Mehregany, R. T. Howe and S. D. Senturia, "Microfabricated structures for the in situ measurement of residual stress, Youngs modulus, and ultimate strain of thin films," Appl. Phys. Lett., 51, pp. 241-243 (1987).

[18] D. Maier-Schneider, A. Ersoy, J . Maibach, D. Schneider and E. Obermeier, "Influence of annealing on elastic properties of LPCVD silicon nitride and LPCVD polysilicon," Sensors and Materials, 7, pp. 121-129 (1995).

[19] J. Pan, P. Lin, F. Maseeh and S. D. Senturia, "Verification of FEM analysis of load-deflection methods for measuring mechanical properties of thin films," Tech. Digest of IEEE Solid-State Sensor and Actuator Workshop, (Hilton Head $S C)$, pp. 70-73 (1990).

[20] J. G. Korvink, "An Implementation of the Adaptive Finite Element Method," (Verlag der Fachvereine, Zurich) (1993).

[21] J. Funk, J. Bühler, J. G. Korvink and H. Baltes, "Thermomechanical Modelling of an Actuated Micromirror," Eurosensors VIII Conf. Book of Abstracts, (LAAS, Toulouse), p.192 (1994).

[22] M. Schneider, J.G. Korvink, H. Baltes, "Magnetostatic Modelling of an Integrated Microconcentrator," Transducers'95 Digest of Technical Papers, in press (1995). 